\title{
Anthropometry based Predictive Equations for Estimating Nigerian Women Percentage Body Fat using Stable Isotope Technique as Reference
}

\author{
O.C. Ogbonna ${ }^{1, *}$, J.O. Ojo ${ }^{2}$, O.O. Oketayo ${ }^{3}$ and G.T. Fadupin ${ }^{1}$ \\ ${ }^{1}$ Department of Human Nutrition, University of Ibadan \\ Ibadan, Nigeria \\ ${ }^{2}$ Department of Physics and Engineering Physics, Obafemi Awolowo University \\ Ile-Ife, Nigeria \\ ${ }^{3}$ Department of Physics, Federal University Oye-Ekiti. \\ Ekiti State, Nigeria \\ *Corresponding author's email: obbypastor [AT] gmail.com
}

\begin{abstract}
ABSTARCT---- The direct measurement of body composition using innovative non-or minimally-invasive technologies such as stable isotope technique, despite its reliability and precision remains a significant challenge. This study was designed to develop predictive equations for estimating the percentage body fat of Nigerian women using isotope dilution (ISD) or gold standard technique (as in IAEA procedure) as reference and validated by bioelectrical impedance technique.The percentage body fats (\% BF) of 173 Nigerian women (aged 18-55 yrs) were estimated using isotope dilution (ISD) or gold standard technique (as in IAEA procedure) as reference and validated by bioelectrical impedance technique. The skinfold thicknesses ( $\mathrm{mm}$ ) such as biceps, triceps, subscapular, suprailiac and abdominal were measured using skinfold langre callipers while body circumferences such as forearm, wrist, waist, abdominal and hip were measured using standard tape rule and in accordance with WHO guidelines. The subjects' percentage body fat (\%BF) ranged from 8.0 to 48.5. Generally, at 0.01 level of significance (Using Statistical Package for Social Scientists (SPSS), higher positive significant correlations were obtained between \% BF by ISD and body circumferences $(r=0.503-0.874)$ than skinfold thicknesses $(r=0.503-0.739)$. Using forward selection regression procedure, three sex-specific based predictive equations for estimating \%BF (incorporating subjects' weight and body circumferences such as hip, abdominal and waist) were developed based on the subjects' ethnic groups (Yoruba, Hausa and Ibos) with high $\boldsymbol{R}^{2}$ $(0.853,0.953 \& 0.867)$ and low Pure errors $(3.316,2.791 \& 2.324)$ respect. These equations provide easy way of measuring percentage body fat and are recommended for use in clinical or epidemiological settings in populations with similar ethnic background and in lieu of isotope dilution method.
\end{abstract}

Keyword--- Anthropometry based predictive equations, percentage body fat, Nigerian women, stable isotope technique

\section{INTRODUCTION}

The quantification of body composition into its fat and fat free components is important in order to describe excesses or deficiencies of fat and Fat Free Mass that have significant associations with the risk or onset of diseases. Increase in the prevalence of body fat within a population often precedes a rise in the occurrence of chronic diseases, such as diabetes mellitus and hypertension (1). Methods often used globally in assessing body composition include the use of Body Mass Index (BMI) which is cheap and non-invasive but non-specific and not an accurate means of measuring body fat, Bioelectric Impedance Analysis (BIA) which is affordable and portable but its accuracy and precision might be affected by subject factors such as races, ethnicity and tends to either underestimate the underweight or overestimate the obese. Other methods include Isotope Dilution (ID) technique, Hydro densitometry (Underwater Weighing), Near Infrared Intereactance (NII), Dual Energy X-ray Absorption (DEXA), Bioelecctrical impedance Analysis, Magnetic Resonance Imaging (MRI), Computed Tomography (CT), Air Displacement (AD) and anthropometry (skinfolds and circumferences). Each with its merits and limitations. Isotope dilution is the gold standard. It is an in-vitro and a non-invasive method. It is a more rigorous method but provides an accurate means of determining body composition. Anthropometric measures for the evaluation of body fat such as hip to waist ratio (HWR) are among the most frequently used for the diagnosis of diseases. They are cost effective and easily performed but cannot thoroughly determine the distribution of body fat (2-4). Similar work was done by Oketayo and Ojo (ㅁ) using Bioelectrical Impedance technique as reference which tends to consistently overestimate lean 
people and underestimate obese people. There is therefore the need to develop simple and a better means of assessing body composition such as predictive equations using mathematical relationships which put into consideration the important sites for fat distribution/storage in the subjects concerned.

In this work, isotope dilution method (often called gold standard technique) was used as a reference (to calibrate the existing bioelectric impedance (BIA) equipment and) to develop anthropometry based predictive equations for estimating body fat, specifically for Nigerian women generally, and on the basis of three major ethnic groups in Nigeria.

\section{MATERIALS AND METHODS}

\subsection{Subject Description and Collection of Anthropometric Data}

A total of one hundred and seventy three (173) healthy Nigerian women (which included 81 Yorubas, 32 Hausas and 60 Igbos) aged 18-55 years were recruited into the study using purposive sampling technique after collecting ethical clearance from Obafemi Awolowo University Teaching Hospital Complex, Ile-Ife Ethical committee. Only those who were willing to participate and those who completed the protocols were finally recruited after explaining the details of the study to them. Subjects' height were measured to the nearest $0.1 \mathrm{~cm}$ by having the subject stand erect, looking straight ahead, against a calibrated wall while the body weight (with minimal clothing and without foot wears) was obtained to the nearest $0.1 \mathrm{~kg}$ using an electronic weighing balance. Body mass index (BMI) was calculated as weight (kg) divided by the square of height $\left(\mathrm{W} / \mathrm{H}^{2}\right)$. Skinfold thicknesses such as biceps, triceps, scapular, subscapular, suprailiac and abdominal were determined using Langre Callipers while circumferences such as forearm, neck, wrist, waist, hip and abdominal were measured (each thrice) using standard tape rule and in accordance with WHO guidelines.

\subsection{Determination of Subject's Body Fat}

The Bioelectrical Impedance (BIA) technique was first used in this study to determine the subjects' percentage body fat and latter validated by the isotope dilution method (the gold standard and a more reliable technique).

2.2.1 BIA Technique: Fat has very high electrical impedance, compared to other tissues. Hence by sending an appropriate alternating current through the body, the total impedance of the body can be obtained. The total impedance increases with BF (using a Tanita body composition analyzer -BF 350). The computer software embedded in the product uses the impedance, subject's gender, height, fitness level, age and weight to determine the percentage body fat based on equation or formula. It determines the $\% \mathrm{BF}$ to the nearest $0.1 \%$.

2.2.2 Isotope Dilution Method: Saliva samples were first collected from the subjects ,first before the administration of an oral dose of $20 \mathrm{mg}$ of sterile $99.9 \%$ deuterium oxide and four hours later (ensuring minimal physical activities)Saliva sample was collected by having the subjects expectorate into a clean plastic $4 \mathrm{~mL}$ tube. In order to remove particulate matters in saliva, the samples were centrifuged at $3000 \mathrm{rpm}$ for 15 minutes . The clear supernatant saliva samples were retrieved with pasteur pipettes, transferred into new tubes and stored at $-20{ }^{\circ} \mathrm{C}$, until analysed. The enrichment of deuterium in the saliva samples were determined by Fourier Transform infrared spectroscopy using the vibrational band produced by deuterated water between 2400 and $2600 \mathrm{~cm}^{-1}$, in the infrared region of the spectrum (ㅁ). This was done at the Multidiscipilinary Research Laboratory of the University of Ibadan, using a special calcium fluoride cell made available by the Central Science Laboratory of the Obafemi Awolowo University, Ile-Ife.

Standards of $\mathrm{D}_{2} \mathrm{O},(0,100,200,400,600,800,1000,1500$ and $2000 \mathrm{ppm})$ were prepared gravimetrically using local drinking (tap) water and the $99.9 \%{ }^{2} \mathrm{H}$ deuterium oxide administered to the subjects according to International Atomic Energy Agency (IAEA) Standard Operating Procedures. The deuterium enrichment $(\mathrm{mg} / \mathrm{kg})$ in the stock and standard solutions were calculated using the formular

$$
\text { Calculated } \mathrm{D}_{2} \mathrm{O} \text { Enrichment }=\frac{\text { Weight of } \mathrm{D}_{2} \mathrm{O}(\mathrm{g})}{\text { Weight of water }(\mathrm{g})} \times 10^{6}(\mathrm{mg} / \mathrm{kg})
$$

The deuterium enrichment $(\mathrm{mg} / \mathrm{kg}$ ) in the stock and standards was also measured using the FTIR spectrometer after the background scanning to ensure that there is no contribution of $\mathrm{CO}_{2}$ to the deuterium peak in the spectrum (without loading the sample). The calculated enrichment was plotted against measured enrichment to obtain the calibration curve. The procedure was repeated twice to ensure proper calibration of the equipment before use.

After obtaining a good calibration curve ( $\mathrm{r}=0.9998)$, the baseline and post-dose saliva samples for each subject were analyzed (20 scans) at $2300-2900 \mathrm{~cm}^{-1}$ at the same absorbance band. 
The total body water (TBW) was calculated using

$$
T B W(K g)=\frac{V_{D}(K g)}{1.041}
$$

while the Fat Free Mass (FFM) was determined using

$$
F F M(K g)=\frac{T B W(K g)}{0.732}
$$

Where $V_{D}$ is the volume of distribution of deuterium (also known as the pool space) bearing in mind that the hydration of FFM in the body is remarkably constant between species. FFM in adults is assumed to be $73.2 \%$ water. Also, $V_{D}$ must be corrected for non-aqueous-isotopic exchange. Non-aqueous isotopic exchange for deuterium is assumed to be $4.1 \%$ of the pool space (ㅁ).

Fat mass (FM) was calculated as the difference between FFM and body weight

$$
\mathrm{FM}(\mathrm{Kg})=\text { Body weight }(\mathrm{Kg})-\mathrm{FFM}(\mathrm{Kg})
$$

Hence, the percentage body fat (also known as FM \%) gives

$$
F M \%=\frac{F M(k g)}{\text { Bodyweight }(K g)} \times 100
$$

(IAEA, 1999)

\subsubsection{Development of Predictive Equations}

In this study, with SPSS 16 forward selection regression procedure was used in the selection of the predictor variables. This procedure involves the selection of predictor variable that has the highest correlation with the response variable to formulate a one-predictor variable equation. A second predictor variable that has the highest $\mathrm{R}$ or $\mathrm{R}^{2}$ value with the response variable is then selected among the remaining predictor variables (to have a two-predictor variable equation). The larger the $R^{2}$ value, the better the equation fits the data. The significance of contribution to the equation is evaluated by the $R^{2}$ value, partial F-test and Standard error $(\mathrm{SE}) . \mathrm{R}^{2}$ is the coefficient of determination and it represents the total variance in the response variable that is explained in the predictor variables in the equation. The procedure is continued until the inclusion of any of the remaining predictor variables does not significantly improve the equation (ㄱ). The Root Mean Square Error (RMSE) was calculated using

$$
R M S E=\sqrt{\frac{\sum(\text { observed }- \text { predicted })^{2}}{n-p-1}}
$$

where $\mathrm{n}$ is the no of observation and $\mathrm{p}$ is the no of predictor variables.

The residual plot is determined using

Re sidual plot $=\frac{\left(Y-Y_{\text {fitted }}\right)}{S D}$

To cross-validate the obtained equations, more female Nigerian subjects (within the same age range) were recruited from the same population. Anthropometric measurements were also carried out on them by the same field assistants using the same procedures and equipment.

The derived equations in this study were applied to these group with the pure error determined using the expression

$$
P E=\sqrt{\sum \frac{Y-\bar{Y}}{N}}
$$


Where $\mathrm{Y}=$ Predicted $\% \mathrm{BF}$,

$\ddot{Y}=$ Observed $\% \mathrm{BF}$ and $\mathrm{N}=$ No of subjects used for cross validation. It measures the performance of a predictive equation on cross validation. For the four groups, both validation and cross validation groups were assigned.

\section{RESULTS}

The descriptive statistics of the subjects' body weight, \% BF, skin-folds and the body circumferences were given as range and mean \pm SD on the basis of the three major ethnic groups in Nigeria and as a group (in Table 1). The degree of correlation among the parameters measured were sought for (at 0.01 and 0.05 level of significance) using Pearson product moment correlation (Table 2). All analyses including formulation of predictive equations were made using SPSS for Windows version 15.0 (SPSS, Chicago, USA). Table 2 shows the degree of correlation among these parameters and the important sites for fat distribution in Nigerian women. The final best predictive sex and ethnicityspecific equations combining the subjects' body weight and circumferences were expressed in Table 3 while Table 4 presents the model summary of the predictive equations developed.

Table 1: Descriptive Statistics of Subjects' \%BF by ISD and anthropometry on the basis of their ethnic groups

\begin{tabular}{lllll}
\hline Parameters & $\begin{array}{l}\text { Mean } \pm \text { SD } \\
\text { All, } \mathrm{n}=173)\end{array}$ & $\begin{array}{l}\text { Mean } \pm \text { SD } \\
\text { Yoruba }(\mathrm{n}=81)\end{array}$ & $\begin{array}{l}\text { Mean } \pm \text { SD } \\
\text { Hausa }(\mathrm{n}=32)\end{array}$ & $\begin{array}{l}\text { Mean } \pm \text { SD } \\
\text { Igbo(n=80) }\end{array}$ \\
\hline Age $(\mathrm{yrs})$ & $34.90 \pm 10.23$ & $33.65 \pm 10.51$ & $38.93 \pm 7.56$ & $35.82 \pm 9.82$ \\
Biceps $(\mathrm{mm})$ & $17.79 \pm 8.59$ & $16.64 \pm 8.49$ & $22.95 \pm 6.80$ & $17.02 \pm 8.76$ \\
Triceps (mm) & $20.56 \pm 8.21$ & $21.00 \pm 8.22$ & $18.37 \pm 6.98$ & $18.68 \pm 7.23$ \\
Subscapular SF (mm) & $20.36 \pm 8.70$ & $20.38 \pm 8.94$ & $22.41 \pm 7.55$ & $18.64 \pm 6.92$ \\
Abdominal SF (mm) & $24.5 \pm 8.02$ & $24.54 \pm 8.41$ & $27.20 \pm 7.10$ & $21.76 \pm 8.49$ \\
Suprailiac SF (mm) & $16.65 \pm 7.04$ & $16.83 \pm 8.45$ & $17.78 \pm 6.74$ & $15.47 \pm 6.90$ \\
Neck CF (cm) & $32.17 \pm 1.91$ & $32.14 \pm 1.98$ & $32.76 \pm 1.75$ & $32.31 \pm 1.93$ \\
Forearm CF (cm) & $28.20 \pm 4.10$ & $25.68 \pm 4.56$ & $29.55 \pm 3.98$ & $29.41 \pm 3.78$ \\
Wrist CF (cm) & $15.74 \pm 1.81$ & $15.73 \pm 1.88$ & $16.09 \pm 1.49$ & $15.87 \pm 1.59$ \\
Abdominal CF (cm) & $83.62 \pm 13.76$ & $82.73 \pm 14.54$ & $89.33 \pm 8.70$ & $86.58 \pm 12.53$ \\
Waist CF (cm) & $90.71 \pm 11.60$ & $90.07 \pm 11.89$ & $94.71 \pm 9.61$ & $90.36 \pm 11.65$ \\
Hip CF(cm) & $99.72 \pm 11.27$ & $99.25 \pm 11.86$ & $103.16 \pm 8.45$ & $99.72 \pm 10.83$ \\
Height (m) & $1.61 \pm 0.06$ & $1.30 \pm 0.02$ & $1.62 \pm 0.05$ & $1.61 \pm 0.06$ \\
Weight (kg) & $66.8 \pm 13.9$ & $64.22 \pm 15.14$ & $69.85 \pm 12.68$ & $66.28 \pm 13.99$ \\
BMI(Kg/m2) & $25.61 \pm 5.0$ & $24.61 \pm 5.49$ & $26.51 \pm 4.39$ & $25.62 \pm 5.11$ \\
\% BF(ISD) & $32.31 \pm 7.61$ & $30.44 \pm 9.16$ & $34.41 \pm 6.21$ & $32.04 \pm 7.41$ \\
\hline
\end{tabular}

Note: $\mathrm{SF}=$ Skinfolds $\mathrm{CF}=$ Circumferences, $\mathrm{ISD}=$ Isotope dilution

Table 2: Correlation Coefficient (R) of the Variables on the basis of Subjects" Ethnic groups (with \%BF (ISD)

\begin{tabular}{lllll}
\hline Parameters & All $(\mathrm{n}=173)$ & Yoruba $(\mathrm{n}=81)$ & Hausa $(\mathrm{n}=32)$ & Igbo $(\mathrm{n}=60)$ \\
\hline Weight $(\mathrm{kg})$ & 0.850 & 0.819 & 0.947 & 0.903 \\
Abdominal CF $(\mathrm{cm})$ & 0.825 & 0.763 & 0.912 & 0.924 \\
Waist CF $(\mathrm{cm})$ & 0.874 & 0.886 & 0.895 & 0.924 \\
Hip CF $(\mathrm{cm})$ & 0.867 & 0.848 & 0.859 & 0.882 \\
Forearm CF $(\mathrm{cm})$ & 0.715 & 0.646 & 0.844 & 0.824 \\
Neck CF $(\mathrm{cm})$ & 0.698 & 0.674 & 0.671 & 0.818 \\
Subscapular SF $(\mathrm{mm})$ & 0.739 & 0.734 & 0.853 & 0.529 \\
Suprailiac SF & 0.659 & 0.580 & 0.792 & 0.797 \\
Abdominal SF & 0.735 & 0.756 & 0.690 & 0.529 \\
Triceps (mm) & 0.698 & 0.706 & 0.794 & 0.750 \\
Biceps $(\mathrm{mm})$ & 0.503 & 0.461 & 0.697 & 0.745 \\
Age $(\mathrm{yrs})$ & 0.538 & 0.598 & 0.456 & 0.503 \\
Height $(\mathrm{m})$ & 0.243 & 0.254 & $0.303 *$ & 0.146 \\
BMI $\left(\mathrm{kg} / \mathrm{m}^{2}\right)$ & 0.812 & 0.772 & 0.911 & 0.836 \\
\hline
\end{tabular}

Note: Asterisked=Not significant at 0.01 Level 


\section{DISCUSSION}

Table 1 briefly summarized the anthropometric characteristics and percentage body fat $(\% \mathrm{BF})$ of the respondents. It was observed that as the age increases, the $\% \mathrm{BF}$ increases as well. This result was in line with the findings of Silver $e$ t al (8). It was also observed that the BMI increased with age which was similar to the findings of Reas et al (ㅁ) .

From Table 2, it is obvious that the fat distribution among the Nigerian women were more noticeable around their abdominal, waist and hip circumferences. Epidemiological studies have increasingly stressed the importance of fat distribution in determining cardiovascular risk status (2). For the Yoruba women, fats were distributed around the waists and the hips, thus conferring on them the" pear shape "morphology with less cardiovascular risk. The Hausa (weight/abdominal circumference) and the Igbo (abdominal and hip circumference) women may be more prone to cardiovascular problems when compared to the Yoruba women by the virtue of their body fat distribution ( 2$)$. They tend to have an 'apple' shape morphology, thus exposing them to risks associated with android obesity (Goon, 2010). This result is similar to the one reported by Goon (2010) where high abdominal central body fat was observed among Nigerian women in Markudi. The implication of this finding is that the Hausa and Igbo women may be susceptible to metabolic disorders and cardiovascular risks. Using ANOVA, it was also observed that there was no significant difference in the fat distribution along the waist line of the three ethnic groups $(\mathrm{p}<0.001)$. However, the Igbo women had more fat along their hip region than the Yoruba and Hausa women; the reason for this is not known but may be due to genetic factor.

The final best predictive sex and ethnicity-specific equations combining the subjects' body weight and circumferences were expressed in Table 3. Natural logarithm was introduced to further improve the $\mathrm{R}^{2}$ values. The $\mathrm{R}$ and $\mathrm{R}^{2}$ values for the developed equations were higher than the existing equations from the literature indicating the fitness of our equations to the data/population considered.

Table 3: Comparison of the developed predictive equations with the existing ones

\begin{tabular}{|c|c|c|c|c|c|}
\hline & Equations & $\begin{array}{l}\text { R- } \\
\text { Value }\end{array}$ & $\mathrm{R}^{2}$ Value & PE & RMSE \\
\hline $\begin{array}{l}\text { Equation } 1 \\
\text { (General, } \mathrm{n}=173 \text { ) }\end{array}$ & $\begin{array}{l}\text { \% } \mathrm{BF}=-223.13+32.57 \mathrm{LnWC}+17.240 \mathrm{LnHC}+4.43 \\
\mathrm{LnWt}+3.36 \mathrm{LnST}\end{array}$ & 0.878 & 0.771 & 3.31 & 3.42 \\
\hline $\begin{array}{l}\text { Equation } 2 \\
\text { (Yoruba, } \mathrm{n}=81 \text { ) }\end{array}$ & $\begin{array}{l}\% \mathrm{BF}=-241.588+38.468 \mathrm{LnWC}+11.233 \mathrm{LnHC}+ \\
11.4951 \mathrm{LnWt}\end{array}$ & 0.831 & 0.690 & 4.36 & 4.67 \\
\hline $\begin{array}{l}\text { Equation } 3 \\
\text { (Hausa, } \mathrm{n}=32 \text { ) }\end{array}$ & $\begin{array}{l}\% \mathrm{BF}=- \\
188.173+17.935 \mathrm{LnWt}+19.050 \mathrm{LnAC}+13.433 \mathrm{LnWC}\end{array}$ & 0.810 & 0.680 & 5.22 & 4.47 \\
\hline $\begin{array}{l}\text { Equation } 4 \\
(\text { Igbo, } n=60)\end{array}$ & $\begin{array}{l}\% \mathrm{BF}=- \\
211.853+31.223 \mathrm{LnHC}+1.497 \mathrm{LnWt}+ \\
\text { 17.718LnAC+4.608LnFC }\end{array}$ & 0.936 & 0.877 & 2.34 & 2.61 \\
\hline $\begin{array}{l}\text { Durnin \& Womersley } \\
-1974 \underline{(10)}\end{array}$ & $\% B F=\left(\frac{4.95}{D}-4.50\right) \times 100$ & 0.800 & 0.643 & 4.31 & 4.46 \\
\hline Brose-1963 (1) & $\% B F=\left(\frac{4.57}{D}-4.142\right) \times 100$ & 0.802 & 0.643 & 4.89 & 5.09 \\
\hline
\end{tabular}

\footnotetext{
$\mathrm{D}=$ Predicted density of the body $(\mathrm{g} / \mathrm{mL}), \quad \mathrm{D}=1.1423-(0.0632 \times \mathrm{L}) \quad \mathrm{WC}=$ Waist circumference HP=Hip circumference $\mathrm{Wt}=$ Weight $\quad \mathrm{AC}=$ Abdominal circumference $\quad \mathrm{ST}=$ Suprailliac thickness $\mathrm{FC}=$ Forearm circumference
} 
The estimates of \% BF from both validation and cross-validation had excellent correlation with the Isotope dilution estimates (Figures 1-4 \& Table 3) indicating a negligible tendency for either underestimation or overestimation of $\% \mathrm{BF}$ in underweight and obese subjects. For the four equations developed (at 95\% confidence interval), the regression sum of the squares were significantly higher than the residual sum of the squares (Table 4) indicating that about $95 \%$ of the variation in percentage body fat is explained by the model.

Table 4: Model Summary for the Predictive Equations

\begin{tabular}{lll}
\hline Predictive equations & $\begin{array}{l}\text { Sum of the Squares (Regression } \\
\text { /Model) }\end{array}$ & $\begin{array}{l}\text { Sum of the Squares } \\
\text { (Residual) }\end{array}$ \\
\hline 1, All $(\mathrm{n}=173)$ & 6315.15 & 1228.60 \\
2. Yoruba $(\mathrm{n}=81)$ & 3112.32 & 869.98 \\
3. Hausa $(\mathrm{n}=32)$ & 1105.58 & 55.07 \\
4. Igbo $(\mathrm{n}=60)$ & 1641.72 & 252.16 \\
\hline
\end{tabular}

\section{Implication for practice/policy}

This work established the fact that body build and anthropometric parameters do vary very significantly from one race to another asides sex, age and other factors. From our findings, the anthropogenic predictive equations developed could be used for body fat estimation in lieu of isotope dilution technique for the subjects.

\section{CONCLUSION}

In this study, the body fat of Nigerian women (aged 18-55 yrs) was estimated using isotope dilution (gold standard) technique. One general and three ethnicity-based predictive equations were also developed using regression analysis (with forward selection regression procedure for choosing predictor variables) and stable isotope dilution technique as reference. The result shows that ethnicity (genetics) is an important factor in fat distribution in the body. This is similar to previous findings by Amy et al (12) that body composition is strongly influenced by genetic and environmental factors. The environmental factors most responsible for the determination of body composition are energy intake and energy expenditure in form of physical activity level. The low SEE, PE, RMSE as well as high $\mathrm{R}^{2}$ and $\mathrm{R}$ values obtained when the equations were applied to cross-validation groups established the precision and reliability of these equations for these group of Nigerian subjects. These equations are easy to use and recommended for use in clinical or epidemiological settings in populations with similar ethnic background in lieu of isotope dilution technique.

\section{ACKNOWLEDGEMENT}

This work was supported by the International Atomic Energy Agency, Vienna (Research Contract RC 13248).

\section{REFERENCES}

[1] Amy, R.D., Arvizu, C.R., Liaine, ., Terence, F.'Relation between Body mass index and body fat in black population sample from Nigeria, Jamaica and United States”, Am. J. Epidemid., 45 (7): pp 1-12. (1997).

[2] Brosek, J, Grande, F., Anderson, T, Keys, A.. "Densitometric Analysis of body composition: revisions of some quantitative assumptions" Ann. NY Acad. Sci., 110, pp113-140, (1963).

[3] Durnin, J.V.A., Womersley, J. "Body fat assessed from total body density and its estimation from skinfold thicknesses: measurement on 481 men and women aged from 16-72 years" British J. Nutr., 32, pp 77-97, (1974).

[4] Goon, DT. "Abdominal body fatness among Nigerian women: a study on the anthropometric index of waist to stature ratio.” Pak. J. Med Sci.., 2010; 26 (3): pp 577-580 (2010).

[5] Guo, S.S, Chumlea, W. "Statistical methods for the development and testing of predictive equations in human composition methods and findings" A.F. Roche, S.B. Haymsfield, Lohman T.G Eds Champaign, pp191-202 ,(1996).

[6] IAEA .Assessment of Body Composition and Total Energy Expenditure in Humans Using Stable Isotope Techniques, IAEA Human Health Series No 3: pp 13-40, (2009). 
[7] Kopelman P 2000. Health risks associated with overweight and obesity, Diabetes care..; 23, pp 1835-1839, (2000).

[8] Lefebvre, H. "Body composition characteristics and body fat distribution in Lean women with polycystic ovary syndrome" Hum.Reprod., 6(6): pp 1255-126, (2011).

[9] Oketayo, O.O and Ojo, J.O "Anthropometric predictive equations for percentage body fat in Nigerian women using bioelectrical impedance as reference" Ife Journal of Science, 2011;13(2)pp 363-369, (2011).

[10] Poultrers, N.R., Khaw, K., Hopwood, B.E "Determinant of blood pressure changes due to urbanization: a longitudinal study” J. Hypertens Suppl., 3: pp 5375-5377, (1985).

[11] Reas. D.L, Jan, F..N, Elisabeth, S., Sandanger, T.I. " "Changes in body mass indexage, gender and socio-economic status among a cohort of Norwegian men and women" (1990-2001), BMC Public Health, 7:pp 269 (2007).

[12] Silver, A.J., Guillen, C.P., Kahl, M.J and Morley J.E. "Effect of aging on body fat" J Am Gerriatr Soc, 4: pp 211-3, (1993).

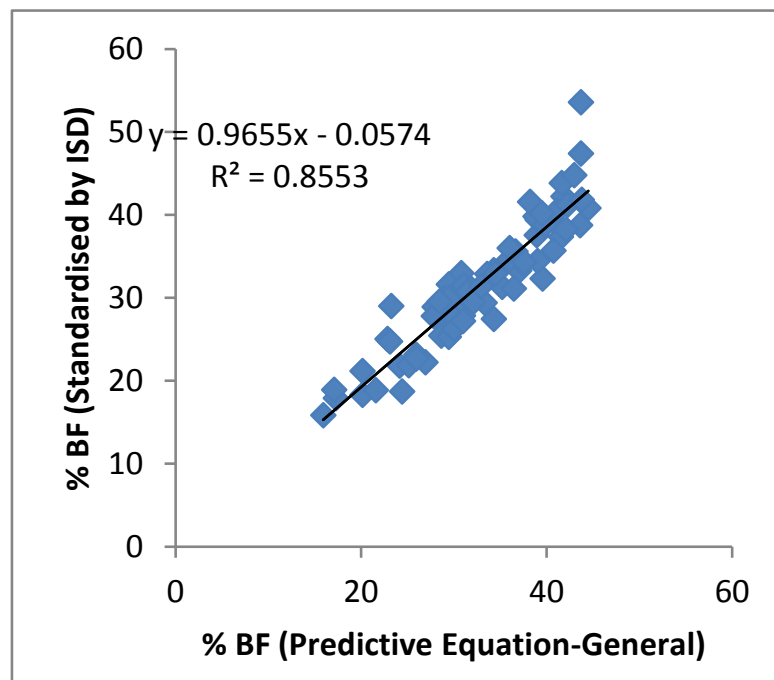

Figure 1: All the subjects

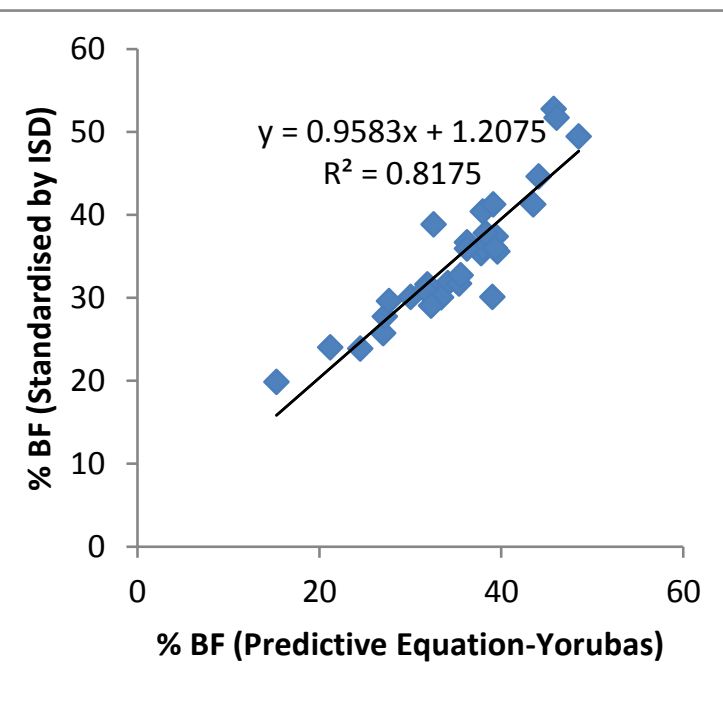

Figure 2: Yoruba

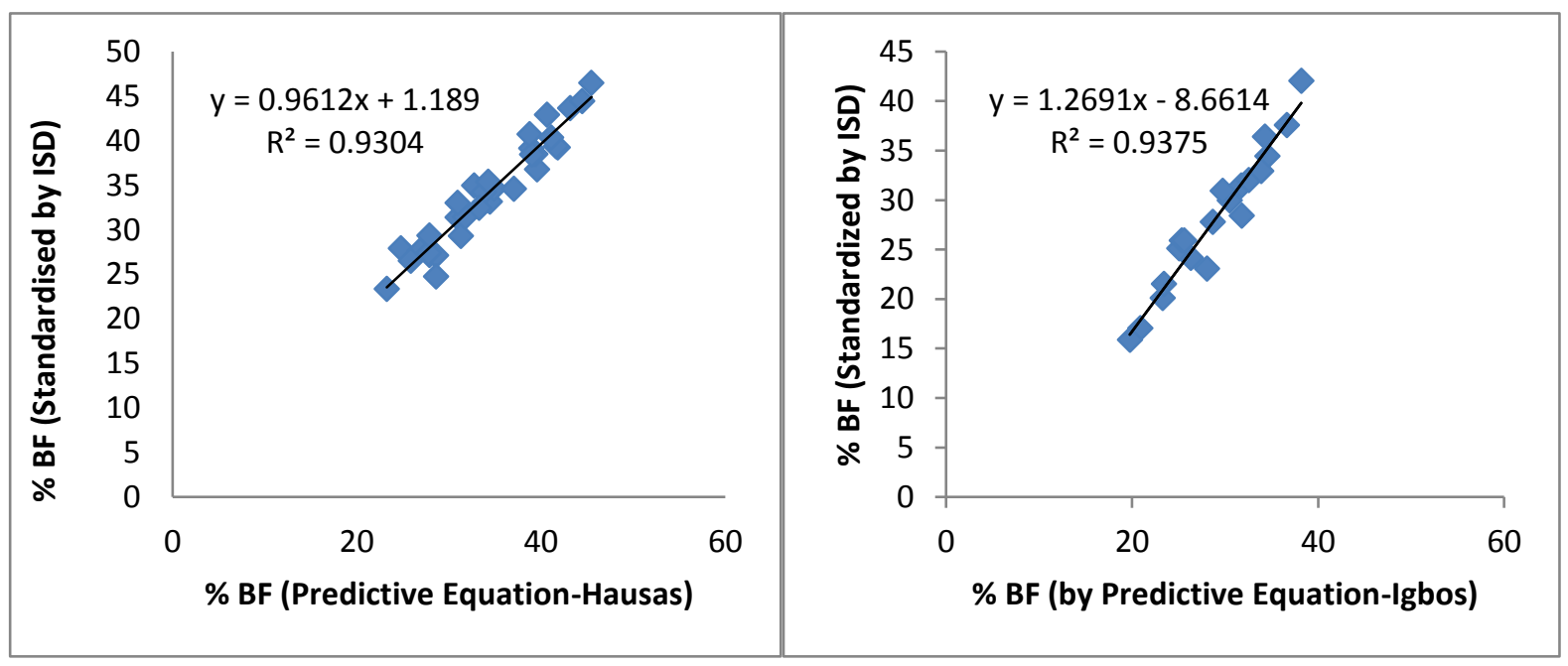

Figure 3: Hausa

Figure 4: Igbo

Figures 1-4: \% BF by BIA (standardized using ISD) against \% BF by predictive equations 